\title{
Detection of Chlamydia trachomatis in urinary samples from women
}

\author{
L-O Svensson, I Mares, S-E Olsson
}

\begin{abstract}
With a mean age of 21 years 197 women at risk for an infection with Chlamydia trachomatis (CT) had a urinary sample $(20 \mathrm{ml}$ first-void urine, minimum 4 hours from prior mictuation) analysed with an enzyme immunoassay (IDEIA-III) for the detection of CT. They also had samples taken from both cervix and urethra for cultivation on McCoy's cells and testing with an enzyme immunoassay (Chlamydiazyme), plus verification of positive samples in the enzyme immunoassay (EIA) with monoclonal antibodies against $C T$. The urethral samples were compared against the urinary samples with regard to sensitivity and specificity in detecting CT. Women with a positive culture for $C T$ and/or a positive verified EIA from either the cervix or the urethra, were regarded as "true" infections with CT. The prevalence of CT was $12 \cdot 2 \%$. The urinary EIA sample had a sensitivity of $84 \%$ whereas the urethral EIA sample had a sensitivity of $57 \%$. The specificity was $98 \%$ and $100 \%$ for the urinary samples, and the urethral samples respectively. It is concluded that the urinary sample is superior to the urethral sample, and that the urinary sample could be used for screening programs, to detect CT among women.
\end{abstract}

\section{Introduction}

To detect Chlamydia trachomatis (CT) infections in women samples for culturing or direct detection are usually taken from cervix and urethra. Some women experience pain when the urethral sample is taken, and some have symptoms of urethritis afterwards. Because of this some young women are reluctant to have samples taken, particularly if they have no symptoms of disease. Women infected with CT are often asymptomatic. ${ }^{1-3}$ Infections with $\mathrm{CT}$ may cause serious complications like salpingitis, increased risk of ectopic pregnancy and infertility. ${ }^{4-6}$ It is therefore

Department of Obstetrics and Gynaecology, and Microbiology, Central Hospital, Västerås, Sweden L-O Svensson, I Mares, S-E Ölsson of great importance to find and treat these asymptomatic women.

Owing to the invasive sampling procedure, screening programmes are difficult to perform. Earlier, urinary specimens had been compared with urethral samples to detect CT using the cell culture technique. The sensitivity for the urinary samples was however not acceptable. ${ }^{7}$ Recently a method has been described to detect $C T$ with enzyme immunoassay (EIA) in urinary samples from men. ${ }^{8-11}$ The aim of this study was to examine women at risk for CT infection with the standard invasive sampling from the cervix and the urethra and to compare with a urinary sample, for the detection of CT.

\section{Subjects and methods}

One hundred and ninety seven women, either pregnant, wanting an abortion or attending the family planning clinic were studied. Their mean age was 21 years (range 16-46). The women were asked to leave a urinary sample $(20 \mathrm{ml}$ first-void urine, minimum 4 hours from prior mictuation). The invasive samples were then taken from urethra and endocervix after the cervix had been wiped clean with a large cotton swab. Samples for cultivation on McCoy's cells were taken with ENT swabs and samples for enzyme immunoassay (Chlamydiazyme, Abbott) were taken with EZE swabs. All the positive samples in the enzyme immunoassays had to be verified in immunofluorescence microscope with CT monoclonal antibodies (Syva, Micro Trak) before they were regarded as truly positive. The urinary samples $(20 \mathrm{ml})$ were centrifuged at $3000 \mathrm{~g}$ for 20 minutes; the sediment was resuspended in $1 \mathrm{ml}$ disruption buffer and analysed with enzyme immunoassay (IDEIA-III, Novo Bio labs) in accordance with the manufacturers instructions. Positive samples were verified as above. The criteria for a CT infection in the urethra or in the cervix, was a positive culture and/or a positive Chlamydiazyme test positively verified with CT monoclonal antibodies.

\section{Results}

Of the 197 women entered in the study $23(11.6 \%)$ had positive endocervical CT cultures. Twenty-four (prevalence $12 \cdot 2 \%$ ) women were positive for CT in one or more of the samples (cultures from cervix and urethra and EIA cervix and urethra). Twenty-three 
women had a positive IDEIA-III urinary test $(11.6 \%)$. Four of those women who were negative in the urinary IDEIA-III test were positive just in the endocervical sample but not in the sample from the urethra. Three of the negative (cultures and EIA) samples from cervix and urethra were positive in the urinary IDEIA-III test only (table 1 ). The sensi-

Table 1 Comparison of urinary IDEIA-III against EIA and/or tissue culture (cervix/urethra) for the detection of $C T . n=197$.

\begin{tabular}{llccr}
\hline & & \multicolumn{2}{l}{$\begin{array}{l}\text { Cervix and/or Urethra } \\
\text { EIA and/or cultivation }\end{array}$} \\
\cline { 3 - 5 } & & Pos & Neg \\
\hline Urine IDEIA-III & Pos & 20 & $3^{\star}$ & 23 \\
& Neg & $44^{\dagger}$ & 170 & 174 \\
& & 24 & 173 & 197
\end{tabular}

Prevalence $12 \cdot 2 \%(24 / 197)$

Specificity $98.3 \%(170 / 173)$

Sensitivity $83.3 \%(20 / 24)$

Positive predictive value $87.0 \%(20 / 23)$

Negative predictive value $97 \cdot 7 \%(170 / 174)$

*One of these three "false positive" was verified as positive with CT monoclonal antibodies. If this sample was considered as "true positive", a change in sensitivity and specificity would be observed (87.5\% and $98.8 \%$ respectively).

†All of these four IDEIA-III negative patients had negative urethral samples.

Table 2 Comparison of the urethra EIA against cervix| urethra culture/EIA for the detection of $C T$.

\begin{tabular}{lllrr}
\hline & & \multicolumn{3}{l}{ Cervix/urethra } \\
\cline { 3 - 5 } & & Pos & Neg \\
\hline Urethra & Pos & 14 & 0 & 14 \\
& Neg & 10 & 173 & 183 \\
& & 24 & 173 & 197
\end{tabular}

Sensitivity $58 \cdot 3 \%(14 / 24)$

Specificity $100 \%(173 / 173)$

Positive predictive value $100 \%(14 / 14)$

Negative predictive value $94.5 \%(173 / 183)$

Table 3 Comparison of urine (IDEIA-III) and urethra (Chlamydiazyme). $(n=197)$

\begin{tabular}{llccr}
\hline & & \multicolumn{3}{l}{ Urethra EIA } \\
\cline { 3 - 5 } & & Pos & Neg \\
\hline Urine IDEIA-III & Pos & 13 & $10^{\star}$ & 23 \\
& Neg & 0 & 174 & 174 \\
& & 13 & 184 & 197
\end{tabular}

Sensitivity $100 \%(13 / 13)$

Specificity $94.6 \%(174 / 184)$

Positive predictive value $56.5 \%(13 / 23)$

Negative predictive value $100 \%(174 / 174)$

*Five of ten "false positive" urinary samples were verified with CT monoclonal antibodies and of these five, four had positive cervical samples. If these verified samples were considered as "true positive", a change in specificity and positive predictive value was observed $(97 \cdot 2 \%$ and $78.3 \%$ respectively). tivity and specificity was $83.5 \%$ and $98.3 \%$ respectively. One of these three false positive results was however positive and verified with monoclonal antibodies against CT. In table 2 , the results from urethra EIA were tested against 24 positive cases (cervix and urethra samples culture and EIA). The sensitivity and specificity was $58.3 \%$ and $100 \%$ respectively. Results from urinary IDEIA-III were tested against the results from the urethral EIA (table 3). Twenty-three were positive in the urinary samples compared with 13 positive urethral samples. This gives a sensitivity for urinary IDEIA-III of $100 \%$ and a specificity of $94.6 \%$. Five of the 10 false positive urinary samples were, however, verified with monoclonal antibodies against CT. If these samples are considered true positives, the specificity would be $97 \cdot 2 \%$.

\section{Discussion}

Analysis of urinary samples for CT with IDEIA-III method compared with samples from the cervix and the urethra, analysed with Chlamydiazyme and tissue culture, had a sensitivity of $83.5 \%$ and a specificity of $98.3 \%$. The corresponding figures for the urethral samples Chlamydiazyme was $56.5 \%$ and $100 \%$ respectively. Regarding the fact that approximately $10 \%$ of women infected with CT have a positive test only from the cervix, these results seem very favourable. Most women infected with CT have few, or no symptoms $\mathrm{s}^{12}$ and are not very likely to seek medical advice. Screening programmes with invasive samples from the urethra or cervix cannot be performed except among special groups of women, for example at family planning clinics etc. The analysis of urinary samples by EIA (IDEIA-III), which had a sensitivity of $83.5 \%$ and a specificity of $98.3 \%$ (table 1) screening programmes among women, seems more realistic. This study also showed that the IDEIA-III urinary test for CT was at least as effective as the urethral EIA. The sensitivity was $100 \%$ and the specificity was $94.6 \%$ (table 3 ). The method of taking invasive samples from the urethra has the disadvantage that it is painful while it is being taken, and for some hours afterwards. Owing to this, some urethral samples are perhaps not taken in an optimal way, and could thus be falsely regarded as negative.$^{13}$ Five of the 10 urinary samples which had corresponding negative urethral samples were verified as positive samples with the CT monoclonal antibody technique. This supports the finding in this study that the urinary sample is more sensitive than the urethral sample in detecting CT. This is also in accordance with preliminary results presented by Mårdh et al. ${ }^{14}$ Chernesky et $a l^{13}$ studied 228 women and found a positive culture rate from invasive samples of $5 \cdot 3 \%$. They also tested IDEIA in urinary sediments and found a sensitivity of $69 \cdot 2 \%$. With the IDEIA-III method used in our study we found a sensitivity of 
$87.5 \%$. There is room for improvement in the verification method. For example the centrifuging of samples would increase the sensitivity. These results suggest that the urethral sample could be substituted by an urinary sample in the routine examinations for the detection of CT. In conclusion we found the urinary IDEIA-III test to be sensitive and specific enough to be used in screening programmes, even in women. The urinary IDEIA-III test was superior to the urethral EIA test in detecting CT.

We express our gratitude to Dr M L Nordström at the Department of Social Medicine, University of Uppsala for valuable advice during this work.

Address for correspondence: Lars-Olof Svensson, Tegelslagarvägen 3, 72348 Västerås, Sweden.

1 Tejls H, Rahm VA, Rosen G, Gnarpe H. Correlation between chlamydia infection and clinical evaluation, vaginal wet smear, and cervical swab test in female adolescents. Am J Obstet Gynecol 1987;157:974-6.

2 Rahm VA, Gnarpe H, Odlind V. Chlamydia trachomatis among sexually active teenage girls: lack of correlation between chlamydial infection, history of the patient and clinical signs of infection. Br J Obstet Gynaecol 1988;95:916-9.

3 Ladany S, Sarov I. Recent advances in Chlamydia trachomatis. Eur J Epidemiol 1985;1:235-56.
4 Osser S, Persson K. Immune response to genital chlamydial infection and influence of Chlamydia pneumoniae (TWAR) antibodies. Eur J Clin Microbiol Infect Dis 1989;8:532-5.

5 Brunham RC, Binns B, McDowell J, Paraskevas M. Chlamydia trachomatis infection in women with ectopic pregnancy. Obstet Gynecol 1986;67:722-6.

6 Svensson L, Mårdh PA, Ahlgren M, Nordenskjöld F. Ectopic pregnancy and antibodies to Chlamydia trachomatis. Fertil Steril 1985;44:313-7.

7 Smith TF, Weed LA. Comparison of urethral swabs, urine, and urinary sediment for isolation of Chlamydia trachomatis. J Clin Micriobiol 1975;2:134-5.

8 Caul EO, Paul ID, Milne JD, Crowley T. Non invasive sampling method for detecting Chlamydia trachomatis. Lancet 1988;ii:1246-7.

9 Brady M, Baker C, Neinstein LS. Asymptomatic Chlamydia trachomatis infections in teenage males. J Adolesc Health Care 1988;9:72-5.

10 Matthews RS, Wise R. Non invasive sampling method for detecting Chlamydia trachomatis. Lancet 1989;i:96.

11 Paul EO, Caul ID. Evaluation of three Chlamydia trachomatis immunoassays with an unbiased noninvasive clinical sample. $J$ Clin Microbiol 1990;28:220-2.

12 Holmes KK. Lower genital tract infections in women: Cystitis/ urethritis, vulvo-vaginitis and cervicitis. In: Holmes $\mathrm{KK}$, Mảrdh PA, Sparling PF, Weisner PJ, eds. Sexually Transmitted Diseases. New York: McGraw Hill, 1984:557-89.

13 Chernesky M, Castriciano S, Sellors J, et al. Detection of Chlamydia trachomatis antigens in urine as an alternative to swabs and cultures. $J$ Infect Dis 1990;161:124-6.

14 Stary A, Bergman S, Mårdh PA. A comparison of voided urine, urethral and cervical samples for the diagnosis of genital chlamydial infections in women attending a STD laboratory, using ELISA tests. International Society for Sexually Trans mitted Diseases Research, Copenhagen 1989: abstract 124. 